Article

\title{
On a Class of Second-Order PDE\&PDI Constrained Robust Modified Optimization Problems
}

\author{
Savin Treanţă $\mathbb{D}$ \\ Department of Applied Mathematics, University Politehnica of Bucharest, 060042 Bucharest, Romania; \\ savin.treanta@upb.ro
}

check for

updates

Citation: Treanţă, S. On a Class of Second-Order PDE\&PDI Constrained Robust Modified Optimization Problems. Mathematics 2021, 9, 1473. https: / / doi.org/10.3390/math9131473

Academic Editor: Francisco Ureña

Received: 22 May 2021

Accepted: 22 June 2021

Published: 23 June 2021

Publisher's Note: MDPI stays neutral with regard to jurisdictional claims in published maps and institutional affiliations.

Copyright: (C) 2021 by the author. Licensee MDPI, Basel, Switzerland. This article is an open access article distributed under the terms and conditions of the Creative Commons Attribution (CC BY) license (https:/ / creativecommons.org/licenses/by/ $4.0 /)$.

\begin{abstract}
In this paper, by using scalar multiple integral cost functionals and the notion of convexity associated with a multiple integral functional driven by an uncertain multi-time controlled second-order Lagrangian, we develop a new mathematical framework on multi-dimensional scalar variational control problems with mixed constraints implying second-order partial differential equations (PDEs) and inequations (PDIs). Concretely, we introduce and investigate an auxiliary (modified) variational control problem, which is much easier to study, and provide some equivalence results by using the notion of a normal weak robust optimal solution.
\end{abstract}

Keywords: uncertain data; controlled second-order Lagrangian; weak robust optimal solution; robust optimality conditions; modified objective function method

\section{Introduction}

As we all know, the partial differential equations (PDEs) and inequations (PDIs) are very important in the study of many processes and phenomena in nature, science and engineering. In this regard, over time, several researchers have taken a particular interest in studying some optimization problems with $\mathrm{ODE}, \mathrm{PDE}$, or isoperimetric constraints. We mention here, for instance, the works of Mititelu [1], Treanţă [2], Olteanu and Treanţă [3], Mititelu and Treanţă [4], and Jayswal et al. [5]. Additionally, since the difficulty of the considered problems was increasing, several auxiliary (modified) optimization problems have been introduced to study the initial problem more easily (see, quite recently, Treanţă [6-9]). Moreover, the complexity of real-life processes and phenomena is very high and often involves uncertainty in initial data. In consequence, many researchers turned their attention to real problems involving higher-order PDEs, isoperimetric restrictions, uncertain data, or a combination thereof. In this respect, the reader is directed to the following research works: Liu and Yuan [10], Jeyakumar et al. [11], Wei et al. [12], Preeti et al. [13], Sun et al. [14], Treanţă [15], Lu et al. [16]. For other different but connected ideas on this subject, the reader is directed to [17-20].

In this paper, motivated and inspired by the above mentioned works, and based on a given class of constrained robust optimization problems, named $(P)$, we introduce and investigate a new class of auxiliary (modified) control problems (which are much easier to study). More precisely, by considering multiple integral cost functionals and mixed (equality and inequality) constraints involving second-order partial derivatives and data uncertainty, we formulate and prove some equivalence results between the two considered classes of control problems. Compared to other research papers in this field, the elements of total novelty included in the paper are represented by the presence of second-order partial derivatives and also by the presence of uncertain data both in the objective functional and in the restrictions. Moreover, the proofs associated with the main results of the paper are presented in an innovative way. In addition, since the mathematical framework developed here is suitable for various approaches and scientific views on complex spatial and temporal behaviors, this paper could be seen as a fundamental work for a large community of researchers in science and engineering. 
The paper is divided as follows. Section 2 introduces the preliminary tools that will be used to describe the problem under study. Section 3 contains the main results of this paper. The first main result, under convexity assumptions of the constraint functionals, formulates the equivalence between the considered second-order PDE\&PDI constrained robust optimization problem $(P)$ and the associated modified problem. The second main result represents the converse of the first main result, assuming only the convexity hyothesis of the objective functional. Section 4 contains the conclusions of the paper and further developments.

\section{Preliminary Tools}

Throughout the paper, we consider the following notations and working hypotheses:

- $\quad \mathbb{R}^{p}, \mathbb{R}^{q}, \mathbb{R}^{r}$ and $\mathbb{R}^{n}$ are Euclidean spaces of dimensions $p, q, r$ and $n$, respectively;

- $\Theta \subset \mathbb{R}^{p}$ is a compact domain, and the point $t=\left(t^{\alpha}\right) \in \Theta$ is a multi-parameter of evolution or multi-time;

- $\quad \mathcal{S}$ is the space of $C^{4}$-class state functions $a=\left(a^{\tau}\right): \Theta \rightarrow \mathbb{R}^{q}$, and $a_{\alpha}:=\frac{\partial a}{\partial t^{\alpha}}, a_{\alpha \beta}:=$ $\frac{\partial^{2} a}{\partial t^{\alpha} \partial t^{\beta}}$ denote the partial speed and partial acceleration, respectively;

- $\quad \mathcal{C}$ is the space of $C^{1}$-class control functions $c=\left(c^{j}\right): \Theta \rightarrow \mathbb{R}^{r}$;

- $d t=d t^{1} \ldots d t^{p}$ is the volume element on $\mathbb{R}^{p} \supset \Theta$;

- $T$ denotes the transpose of a vector;

- $\quad$ For two vectors $x, y \in \mathbb{R}^{n}$, we use the following convention for inequalities and equalities:

(i) $x<y \Leftrightarrow x_{i}<y_{i}, \forall i=\overline{1, n}$;

(ii) $x=y \Leftrightarrow x_{i}=y_{i}, \forall i=\overline{1, n}$;

(iii) $x \leqq y \Leftrightarrow x_{i} \leq y_{i}, \forall i=\overline{1, n}$;

(iv) $x \leq y \Leftrightarrow x_{i} \leq y_{i}, \forall i=\overline{1, n}$ and $x_{i}<y_{i}$ for some $i$.

The second-order PDE\&PDI constrained controlled scalar optimization problem with uncertainty in the objective and constraint functionals is formulated as follows:

$$
\min _{(a(\cdot), c(\cdot))} \int_{\Theta} f\left(t, a(t), a_{\sigma}(t), a_{\alpha \beta}(t), c(t), w\right) d t
$$

subject to

$$
\begin{gathered}
g\left(t, a(t), a_{\sigma}(t), a_{\alpha \beta}(t), c(t), u\right) \leqq 0, \quad t \in \Theta \\
h\left(t, a(t), a_{\sigma}(t), a_{\alpha \beta}(t), c(t), v\right)=0, \quad t \in \Theta \\
a\left(t_{0}\right)=a_{0}, a\left(t_{1}\right)=a_{1}, a_{\sigma}\left(t_{0}\right)=a_{\sigma 0}, a_{\sigma}\left(t_{1}\right)=a_{\sigma 1},
\end{gathered}
$$

or

$$
\left.a(t)\right|_{\partial \Theta}=\text { given, }\left.\quad a_{\sigma}(t)\right|_{\partial \Theta}=\text { given, }
$$

where $f: J^{2}\left(\Theta, \mathbb{R}^{q}\right) \times \mathcal{C} \times W \rightarrow \mathbb{R}, g=\left(g_{1}, \ldots, g_{m}\right)=\left(g_{l}\right): J^{2}\left(\Theta, \mathbb{R}^{q}\right) \times \mathcal{C} \times U_{l} \rightarrow$ $\mathbb{R}^{m}, l=\overline{1, m}, h=\left(h_{1}, \ldots, h_{n}\right)=\left(h_{\zeta}\right): J^{2}\left(\Theta, \mathbb{R}^{q}\right) \times \mathcal{C} \times V_{\zeta} \rightarrow \mathbb{R}^{n}, \zeta=\overline{1, n}$, are $C^{3}$-class functionals, $w, u=\left(u_{l}\right)$ and $v=\left(v_{\zeta}\right)$ are the uncertain parameters for some convex compact subsets $W \subset \mathbb{R}, U=\left(U_{l}\right) \subset \mathbb{R}^{m}$ and $V=\left(V_{\zeta}\right) \subset \mathbb{R}^{n}$, respectively, and $J^{2}\left(\Theta, \mathbb{R}^{q}\right)$ denotes the second-order jet bundle associated to $\Theta$ and $\mathbb{R}^{q}$.

The associated robust counterpart of the aforementioned scalar optimization problem $(P)$ is defined as:

$$
\min _{(a(\cdot), c(\cdot))} \int_{\Theta} \max _{w \in W} f\left(t, a(t), a_{\sigma}(t), a_{\alpha \beta}(t), c(t), w\right) d t
$$


subject to

$$
\begin{aligned}
& g\left(t, a(t), a_{\sigma}(t), a_{\alpha \beta}(t), c(t), u\right) \leqq 0, \quad t \in \Theta, \forall u \in U \\
& h\left(t, a(t), a_{\sigma}(t), a_{\alpha \beta}(t), c(t), v\right)=0, \quad t \in \Theta, \forall v \in V \\
& a\left(t_{0}\right)=a_{0}, a\left(t_{1}\right)=a_{1}, a_{\sigma}\left(t_{0}\right)=a_{\sigma 0}, a_{\sigma}\left(t_{1}\right)=a_{\sigma 1},
\end{aligned}
$$

or

$$
\left.a(t)\right|_{\partial \Theta}=\text { given, }\left.\quad a_{\sigma}(t)\right|_{\partial \Theta}=\text { given } .
$$

Furthermore, denoted by

$$
\begin{gathered}
D=\left\{(a, c) \in \mathcal{S} \times \mathcal{C}: g\left(t, a(t), a_{\sigma}(t), a_{\alpha \beta}(t), c(t), u\right) \leqq 0,\right. \\
h\left(t, a(t), a_{\sigma}(t), a_{\alpha \beta}(t), c(t), v\right)=0, a\left(t_{0}\right)=a_{0}, a\left(t_{1}\right)=a_{1}, \\
\left.a_{\sigma}\left(t_{0}\right)=a_{\sigma 0}, a_{\sigma}\left(t_{1}\right)=a_{\sigma 1}, t \in \Theta, u \in U, v \in V\right\}
\end{gathered}
$$

the set of all feasible solutions in $(R P)$ and we say that it is the robust feasible solution set to the problem $(P)$.

From now on, to simplify our presentation, we introduce the following notation: $\pi=\left(t, a(t), a_{\sigma}(t), a_{\alpha \beta}(t), c(t)\right)$.

The first-order partial derivatives associated with $f$ are defined as

$$
f_{a}=\left(\frac{\partial f}{\partial a^{1}}, \cdots, \frac{\partial f}{\partial a^{q}}\right), \quad f_{c}=\left(\frac{\partial f}{\partial c^{1}}, \cdots, \frac{\partial f}{\partial c^{r}}\right) .
$$

Similarly, we have $g_{a}$ and $g_{c}$ using matrices with $m$ rows and $h_{a}$ and $h_{c}$ using matrices with $n$ rows.

In the following, we introduce the notion of a weak robust optimal solution for the considered class of constrained optimization problems. This concept will be used to formulate the associated robust necessary optimality conditions and the main results included in the present paper.

Definition 1. A robust feasible solution $(\bar{a}, \bar{c}) \in D$ is said to be a weak robust optimal solution to the multi-dimensional scalar optimization problem $(P)$ if there does not exist another point $(a, c) \in D$ such that

$$
\int_{\Theta} \max _{w \in W} f(\pi, w) d t<\int_{\Theta} \max _{w \in W} f(\bar{\pi}, w) d t .
$$

To formulate the concept of convexity and the robust optimality conditions associated with the aforementioned controlled optimization problem, we shall use the Saunders's multi-index notation (see Saunders [21], Treanţă [22]).

Definition 2. A multiple integral functional (driven by an uncertain multi-time controlled secondorder Lagrangian)

$$
F(a, c, \bar{w})=\int_{\Theta} f\left(t, a(t), a_{\sigma}(t), a_{\alpha \beta}(t), c(t), \bar{w}\right) d t=\int_{\Theta} f(\pi, \bar{w}) d t
$$

is said to be convex at $(\bar{a}, \bar{c}) \in \mathcal{S} \times \mathcal{C}$ if the following inequality

$$
\begin{gathered}
F(a, c, \bar{w})-F(\bar{a}, \bar{c}, \bar{w}) \geq \int_{\Theta} f_{a}(\bar{\pi}, \bar{w})[a(t)-\bar{a}(t)] d t \\
+\int_{\Theta} f_{a_{\sigma}}(\bar{\pi}, \bar{w})\left[a_{\sigma}(t)-\bar{a}_{\sigma}(t)\right] d t \\
+\frac{1}{n(\alpha, \beta)} \int_{\Theta} f_{a_{\alpha \beta}}(\bar{\pi}, \bar{w})\left[a_{\alpha \beta}(t)-\bar{a}_{\alpha \beta}(t)\right] d t
\end{gathered}
$$




$$
+\int_{\Theta} f_{c}(\bar{\pi}, \bar{w})[c(t)-\bar{c}(t)] d t
$$

holds for all $(a, c) \in \mathcal{S} \times \mathcal{C}$.

In the following, according to Treanţă [22], we present the robust necessary optimality conditions for the scalar optimization problem $(P)$.

Theorem 1. Let $(\bar{a}, \bar{c}) \in D$ be a weak robust optimal solution to the problem $(P)$ and $\max _{w \in W} f(\pi, w)=f(\pi, \bar{w})$. Then, then there exist the scalar $\bar{\mu} \in \mathbb{R}$, the piecewise smooth functions $\bar{v}=\left(\bar{v}_{l}(t)\right) \in \mathbb{R}_{+}^{m}, \bar{\gamma}=\left(\bar{\gamma}_{\zeta}(t)\right) \in \mathbb{R}^{n}$, and the uncertainty parameters $\bar{u} \in U$ and $\bar{v} \in V$, such that the following conditions

$$
\begin{gathered}
\bar{\mu} f_{a}(\bar{\pi}, \bar{w})+\bar{v}^{T} g_{a}(\bar{\pi}, \bar{u})+\bar{\gamma}^{T} h_{a}(\bar{\pi}, \bar{v}) \\
-D_{\sigma}\left[\bar{\mu} f_{a_{\sigma}}(\bar{\pi}, \bar{w})+\bar{v}^{T} g_{a_{\sigma}}(\bar{\pi}, \bar{u})+\bar{\gamma}^{T} h_{a_{\sigma}}(\bar{\pi}, \bar{v})\right] \\
+\frac{1}{n(\alpha, \beta)} D_{\alpha \beta}^{2}\left[\bar{\mu} f_{a_{\alpha \beta}}(\bar{\pi}, \bar{w})+\bar{v}^{T} g_{a_{\alpha \beta}}(\bar{\pi}, \bar{u})+\bar{\gamma}^{T} h_{a_{\alpha \beta}}(\bar{\pi}, \bar{v})\right]=0, \\
\bar{\mu} f_{c}(\bar{\pi}, \bar{w})+\bar{v}^{T} g_{c}(\bar{\pi}, \bar{u})+\bar{\gamma}^{T} h_{c}(\bar{\pi}, \bar{v})=0, \\
\bar{v}^{T} g(\bar{\pi}, \bar{u})=0, \bar{v} \geqq 0, \\
\bar{\mu} \geq 0
\end{gathered}
$$

hold for all $t \in \Theta$, except at discontinuities.

Remark 1. The conditions (1)-(4) are known as robust necessary optimality conditions for the multi-dimensional scalar optimization problem $(P)$.

Definition 3. A robust feasible solution $(\bar{a}, \bar{c})$ is said to be a normal weak robust optimal solution to the problem $(P)$ if $\bar{\mu}>0$ in Theorem 1 . Without loss of generality, we can consider $\bar{\mu}=1$.

\section{The Associated Modified Optimization Problem}

In this section, we use the modified objective function method to reduce the complexity associated with the considered multi-dimensional scalar optimization problem $(P)$. In this regard, let $(\bar{a}, \bar{c})$ be an arbitrary given robust feasible solution to the multi-dimensional scalar optimization problem $(P)$. The modified multi-dimensional scalar optimization problem associated with the original variational control problem $(P)$ is defined as:

$$
\begin{gathered}
(P)_{(\bar{a}, \bar{c})} \min _{(a(\cdot), c(\cdot))} \int_{\Theta}\left\{f_{a}(\bar{\pi}, w)(a(t)-\bar{a}(t))+f_{a_{\sigma}}(\bar{\pi}, w)\left(a_{\sigma}(t)-\bar{a}_{\sigma}(t)\right)\right. \\
\left.\quad+\frac{1}{n(\alpha, \beta)} f_{a_{\alpha \beta}}(\bar{\pi}, w)\left(a_{\alpha \beta}(t)-\bar{a}_{\alpha \beta}(t)\right)+f_{c}(\bar{\pi}, w)(c(t)-\bar{c}(t))\right\} d t
\end{gathered}
$$

subject to

$$
\begin{gathered}
g(\pi, u) \leqq 0, \quad t \in \Theta \\
h(\pi, v)=0, \quad t \in \Theta \\
a\left(t_{0}\right)=a_{0}, a\left(t_{1}\right)=a_{1}, a_{\sigma}\left(t_{0}\right)=a_{\sigma 0}, a_{\sigma}\left(t_{1}\right)=a_{\sigma 1},
\end{gathered}
$$

or

$$
\left.a(t)\right|_{\partial \Theta}=\text { given, }\left.a_{\sigma}(t)\right|_{\partial \Theta}=\text { given, }
$$

where the functionals $f, g$ and $h$ are given as in the original variational control problem $(P)$. 
The associated robust counterpart of the multi-dimensional scalar optimization prob$\operatorname{lem}(P)_{(\bar{a}, \bar{c})}$ is defined as:

$$
\begin{gathered}
(R P)_{(\bar{a}, \bar{c})} \min _{(a(\cdot), c(\cdot))} \int_{\Theta} \max _{w \in W}\left\{f_{a}(\bar{\pi}, w)(a(t)-\bar{a}(t))+f_{a_{\sigma}}(\bar{\pi}, w)\left(a_{\sigma}(t)-\bar{a}_{\sigma}(t)\right)\right. \\
\left.+\frac{1}{n(\alpha, \beta)} f_{a_{\alpha \beta}}(\bar{\pi}, w)\left(a_{\alpha \beta}(t)-\bar{a}_{\alpha \beta}(t)\right)+f_{c}(\bar{\pi}, w)(c(t)-\bar{c}(t))\right\} d t
\end{gathered}
$$

subject to

$$
\begin{gathered}
g(\pi, u) \leqq 0, \quad t \in \Theta, \forall u \in U \\
h(\pi, v)=0, \quad t \in \Theta, \forall v \in V \\
a\left(t_{0}\right)=a_{0}, a\left(t_{1}\right)=a_{1}, a_{\sigma}\left(t_{0}\right)=a_{\sigma 0}, a_{\sigma}\left(t_{1}\right)=a_{\sigma 1},
\end{gathered}
$$

or

$$
\left.a(t)\right|_{\partial \Theta}=\text { given, }\left.\quad a_{\sigma}(t)\right|_{\partial \Theta}=\text { given } .
$$

Remark 2. Let us remark that the robust feasible solution set to the problem $(P)_{(\bar{a}, \bar{c})}$ is the same as in the problem $(P)$, and, in consequence, it is also denoted by $D$.

Definition 4. A point $(\hat{a}, \hat{c}) \in D$ is said to be a weak robust optimal solution to the modified multidimensional scalar optimization problem $(P)_{(\bar{a}, \bar{c})}$ if there does not exist another point $(a, c) \in D$ such that

$$
\begin{aligned}
& \int_{\Theta} \max _{w \in W}\left[f_{a}(\bar{\pi}, w)(a-\bar{a})+f_{a_{\sigma}}(\bar{\pi}, w)\left(a_{\sigma}-\bar{a}_{\sigma}\right)\right. \\
+ & \left.\frac{1}{n(\alpha, \beta)} f_{a_{\alpha \beta}}(\bar{\pi}, w)\left(a_{\alpha \beta}-\bar{a}_{\alpha \beta}\right)+f_{c}(\bar{\pi}, w)(c-\bar{c})\right] d t \\
< & \int_{\Theta} \max _{w \in W}\left[f_{a}(\bar{\pi}, w)(\hat{a}-\bar{a})+f_{a_{\sigma}}(\bar{\pi}, w)\left(\hat{a}_{\sigma}-\bar{a}_{\sigma}\right)\right. \\
+ & \left.\frac{1}{n(\alpha, \beta)} f_{a_{\alpha \beta}}(\bar{\pi}, w)\left(\hat{a}_{\alpha \beta}-\bar{a}_{\alpha \beta}\right)+f_{c}(\bar{\pi}, w)(\hat{c}-\bar{c})\right] d t .
\end{aligned}
$$

In the following, we establish some equivalence results between the problems $(P)$ and $(P)_{(\bar{a}, \bar{c})}$, under some appropriate convexity assumptions.

Theorem 2. Let $(\bar{a}, \bar{c})$ be a normal weak robust optimal solution to the scalar optimization problem $(P)$. Furthermore, assume that $\int_{\Theta} \bar{v}^{T} g(\pi, \bar{u}) d t, \int_{\Theta} \bar{\gamma}^{T} h(\pi, \bar{v}) d t$ are convex at $(\bar{a}, \bar{c})$. Then, $(\bar{a}, \bar{c})$ is also a weak robust optimal solution to the modified multi-dimensional scalar optimization problem $(P)_{(\bar{a}, \bar{c})}$.

Proof. By hypothesis, the relations (1)-(4), with $\bar{\mu}=1$, are fulfilled for all $t \in \Theta$, except at discontinuities. Hence, the conditions (1) and (2) yield

$$
\begin{gathered}
\int_{\Theta}(\tilde{a}-\bar{a})\left\{f_{a}(\bar{\pi}, \bar{w})+\bar{v}^{T} g_{a}(\bar{\pi}, \bar{u})+\bar{\gamma}^{T} h_{a}(\bar{\pi}, \bar{v})\right. \\
-D_{\sigma}\left[f_{a_{\sigma}}(\bar{\pi}, \bar{w})+\bar{v}^{T} g_{a_{\sigma}}(\bar{\pi}, \bar{u})+\bar{\gamma}^{T} h_{a_{\sigma}}(\bar{\pi}, \bar{v})\right] \\
\left.+\frac{1}{n(\alpha, \beta)} D_{\alpha \beta}^{2}\left[f_{a_{\alpha \beta}}(\bar{\pi}, \bar{w})+\bar{v}^{T} g_{a_{\alpha \beta}}(\bar{\pi}, \bar{u})+\bar{\gamma}^{T} h_{a_{\alpha \beta}}(\bar{\pi}, \bar{v})\right]\right\} d t \\
+\int_{\Theta}(\tilde{c}-\bar{c})\left\{f_{c}(\bar{\pi}, \bar{w})+\bar{v}^{T} g_{c}(\bar{\pi}, \bar{u})+\bar{\gamma}^{T} h_{c}(\bar{\pi}, \bar{v})\right\} d t \\
=\int_{\Theta}\left[(\tilde{a}-\bar{a})\left\{f_{a}(\bar{\pi}, \bar{w})+\bar{v}^{T} g_{a}(\bar{\pi}, \bar{u})+\bar{\gamma}^{T} h_{a}(\bar{\pi}, \bar{v})\right\}\right.
\end{gathered}
$$




$$
\begin{gathered}
+\left(\tilde{a}_{\sigma}-\bar{a}_{\sigma}\right)\left\{f_{a_{\sigma}}(\bar{\pi}, \bar{w})+\bar{v}^{T} g_{a_{\sigma}}(\bar{\pi}, \bar{u})+\bar{\gamma}^{T} h_{a_{\sigma}}(\bar{\pi}, \bar{v})\right\} \\
\left.+\frac{1}{n(\alpha, \beta)}\left(\tilde{a}_{\alpha \beta}-\bar{a}_{\alpha \beta}\right)\left\{f_{a_{\alpha \beta}}(\bar{\pi}, \bar{w})+\bar{v}^{T} g_{a_{\alpha \beta}}(\bar{\pi}, \bar{u})+\bar{\gamma}^{T} h_{a_{\alpha \beta}}(\bar{\pi}, \bar{v})\right\}\right] d t \\
+\int_{\Theta}(\tilde{c}-\bar{c})\left\{f_{c}(\bar{\pi}, \bar{w})+\bar{v}^{T} g_{c}(\bar{\pi}, \bar{u})+\bar{\gamma}^{T} h_{c}(\bar{\pi}, \bar{v})\right\} d t=0,
\end{gathered}
$$

where we used the formula of integration by parts, the divergence formula and the boundary conditions formulated in the considered problem.

Now, let us proceed by contradiction and consider $(\bar{a}, \bar{c})$ is not a weak robust optimal solution to the modified multi-dimensional scalar optimization problem $(P)_{(\bar{a}, \bar{c})}$. Then, there exists $(\tilde{a}, \tilde{c}) \in D$ such that

$$
\begin{aligned}
& \int_{\Theta} \max _{w \in W}\left[f_{a}(\bar{\pi}, w)(\tilde{a}-\bar{a})+f_{a_{\sigma}}(\bar{\pi}, w)\left(\tilde{a}_{\sigma}-\bar{a}_{\sigma}\right)\right. \\
+ & \left.\frac{1}{n(\alpha, \beta)} f_{a_{\alpha \beta}}(\bar{\pi}, w)\left(\tilde{a}_{\alpha \beta}-\bar{a}_{\alpha \beta}\right)+f_{c}(\bar{\pi}, w)(\tilde{c}-\bar{c})\right] d t \\
< & \int_{\Theta} \max _{w \in W}\left[f_{a}(\bar{\pi}, w)(\bar{a}-\bar{a})+f_{a_{\sigma}}(\bar{\pi}, w)\left(\bar{a}_{\sigma}-\bar{a}_{\sigma}\right)\right. \\
+ & \left.\frac{1}{n(\alpha, \beta)} f_{a_{\alpha \beta}}(\bar{\pi}, w)\left(\bar{a}_{\alpha \beta}-\bar{a}_{\alpha \beta}\right)+f_{c}(\bar{\pi}, w)(\bar{c}-\bar{c})\right] d t .
\end{aligned}
$$

Since $\max _{w \in W} f(\pi, w)=f(\pi, \bar{w})$, we obtain

$$
\begin{gathered}
\int_{\Theta}\left[f_{a}(\bar{\pi}, \bar{w})(\tilde{a}-\bar{a})+f_{a_{\sigma}}(\bar{\pi}, \bar{w})\left(\tilde{a}_{\sigma}-\bar{a}_{\sigma}\right)\right. \\
\left.+\frac{1}{n(\alpha, \beta)} f_{a_{\alpha \beta}}(\bar{\pi}, \bar{w})\left(\tilde{a}_{\alpha \beta}-\bar{a}_{\alpha \beta}\right)+f_{c}(\bar{\pi}, \bar{w})(\tilde{c}-\bar{c})\right] d t \\
<\int_{\Theta}\left[f_{a}(\bar{\pi}, \bar{w})(\bar{a}-\bar{a})+f_{a_{\sigma}}(\bar{\pi}, \bar{w})\left(\bar{a}_{\sigma}-\bar{a}_{\sigma}\right)\right. \\
\left.+\frac{1}{n(\alpha, \beta)} f_{a_{\alpha \beta}}(\bar{\pi}, \bar{w})\left(\bar{a}_{\alpha \beta}-\bar{a}_{\alpha \beta}\right)+f_{c}(\bar{\pi}, \bar{w})(\bar{c}-\bar{c})\right] d t,
\end{gathered}
$$

equivalent with

$$
\begin{gathered}
\int_{\Theta}\left[f_{a}(\bar{\pi}, \bar{w})(\tilde{a}-\bar{a})+f_{a_{\sigma}}(\bar{\pi}, \bar{w})\left(\tilde{a}_{\sigma}-\bar{a}_{\sigma}\right)\right. \\
\left.+\frac{1}{n(\alpha, \beta)} f_{a_{\alpha \beta}}(\bar{\pi}, \bar{w})\left(\tilde{a}_{\alpha \beta}-\bar{a}_{\alpha \beta}\right)+f_{c}(\bar{\pi}, \bar{w})(\tilde{c}-\bar{c})\right] d t<0 .
\end{gathered}
$$

The robust feasibility of $(\tilde{a}, \tilde{c})$ in the problem $(P)$ and the robust necessary efficiency condition (3) yield

$$
\int_{\Theta} \bar{v}^{T} g(\tilde{\pi}, \bar{u}) d t-\int_{\Theta} \bar{v}^{T} g(\bar{\pi}, \bar{u}) d t \leq 0 .
$$

By hypothesis, since $\int_{\Theta} \bar{v}^{T} g(\pi, \bar{u}) d t$ is convex at $(\bar{a}, \bar{c})$, we obtain

$$
\begin{gathered}
\int_{\Theta}\left\{\bar{v}^{T} g(\tilde{\pi}, \bar{u})-\bar{v}^{T} g(\bar{\pi}, \bar{u})\right\} d t \geq \int_{\Theta}(\tilde{a}-\bar{a}) \bar{v}^{T} g_{a}(\bar{\pi}, \bar{u}) d t \\
+\int_{\Theta}\left(\tilde{a}_{\sigma}-\bar{a}_{\sigma}\right) \bar{v}^{T} g_{a_{\sigma}}(\bar{\pi}, \bar{u}) d t \\
+\frac{1}{n(\alpha, \beta)} \int_{\Theta}\left(\tilde{a}_{\alpha \beta}-\bar{a}_{\alpha \beta}\right) \bar{v}^{T} g_{a_{\alpha \beta}}(\bar{\pi}, \bar{u}) d t+\int_{\Theta}(\tilde{c}-\bar{c}) \bar{v}^{T} g_{c}(\bar{\pi}, \bar{u}) d t .
\end{gathered}
$$


The above inequality together with relation (7) yield

$$
\begin{gathered}
\int_{\Theta}(\tilde{a}-\bar{a}) \bar{v}^{T} g_{a}(\bar{\pi}, \bar{u}) d t+\int_{\Theta}\left(\tilde{a}_{\sigma}-\bar{a}_{\sigma}\right) \bar{v}^{T} g_{a_{\sigma}}(\bar{\pi}, \bar{u}) d t \\
+\frac{1}{n(\alpha, \beta)} \int_{\Theta}\left(\tilde{a}_{\alpha \beta}-\bar{a}_{\alpha \beta}\right) \bar{v}^{T} g_{a_{\alpha \beta}}(\bar{\pi}, \bar{u}) d t+\int_{\Theta}(\tilde{c}-\bar{c}) \bar{v}^{T} g_{c}(\bar{\pi}, \bar{u}) d t \leq 0 .
\end{gathered}
$$

Furthermore, from the assumption, $\int_{\Theta} \bar{\gamma}^{T} h(\pi, \bar{v}) d t$ is convex at $(\bar{a}, \bar{c})$ and the robust feasibility of $(\tilde{a}, \tilde{c})$ and $(\bar{a}, \bar{c})$ in the multi-dimensional scalar optimization problem $(P)$, we obtain

$$
\begin{gathered}
\int_{\Theta}(\tilde{a}-\bar{a}) \bar{\gamma}^{T} h_{a}(\bar{\pi}, \bar{v}) d t+\int_{\Theta}\left(\tilde{a}_{\sigma}-\bar{a}_{\sigma}\right) \bar{\gamma}^{T} h_{a_{\sigma}}(\bar{\pi}, \bar{v}) d t \\
+\frac{1}{n(\alpha, \beta)} \int_{\Theta}\left(\tilde{a}_{\alpha \beta}-\bar{a}_{\alpha \beta}\right) \bar{\gamma}^{T} h_{a_{\alpha \beta}}(\bar{\pi}, \bar{v}) d t+\int_{\Theta}(\tilde{c}-\bar{c}) \bar{\gamma}^{T} h_{c}(\bar{\pi}, \bar{v}) d t \leq 0 .
\end{gathered}
$$

On combining the inequalities (6), (8) and (9), we obtain

$$
\begin{gathered}
\int_{\Theta}\left[(\tilde{a}-\bar{a})\left\{f_{a}(\bar{\pi}, \bar{w})+\bar{v}^{T} g_{a}(\bar{\pi}, \bar{u})+\bar{\gamma}^{T} h_{a}(\bar{\pi}, \bar{v})\right\}\right. \\
+\left(\tilde{a}_{\sigma}-\bar{a}_{\sigma}\right)\left\{f_{a_{\sigma}}(\bar{\pi}, \bar{w})+\bar{v}^{T} g_{a_{\sigma}}(\bar{\pi}, \bar{u})+\bar{\gamma}^{T} h_{a_{\sigma}}(\bar{\pi}, \bar{v})\right\} \\
\left.+\frac{1}{n(\alpha, \beta)}\left(\tilde{a}_{\alpha \beta}-\bar{a}_{\alpha \beta}\right)\left\{f_{a_{\alpha \beta}}(\bar{\pi}, \bar{w})+\bar{v}^{T} g_{a_{\alpha \beta}}(\bar{\pi}, \bar{u})+\bar{\gamma}^{T} h_{a_{\alpha \beta}}(\bar{\pi}, \bar{v})\right\}\right] d t \\
+\int_{\Theta}(\tilde{c}-\bar{c})\left\{f_{c}(\bar{\pi}, \bar{w})+\bar{v}^{T} g_{c}(\bar{\pi}, \bar{u})+\bar{\gamma}^{T} h_{c}(\bar{\pi}, \bar{v})\right\} d t<0,
\end{gathered}
$$

which contradicts the relation (5). Consequently, $(\bar{a}, \bar{c})$ is a weak robust optimal solution to the problem $(P)_{(\bar{a}, \bar{c})}$, and this completes the proof.

The next result represents the reciprocal of the above theorem.

Theorem 3. Let $(\bar{a}, \bar{c})$ be a weak robust optimal solution to the modified scalar optimization problem $(P)_{(\bar{a}, \bar{c})}$. Furthermore, assume that $\max _{w \in W} f(\pi, w)=f(\pi, \bar{w})$ and the functional $\int_{\Theta} f(\pi, \bar{w}) d t$ is convex at $(\bar{a}, \bar{c})$. Then, $(\bar{a}, \bar{c})$ is a weak robust optimal solution to the multi-dimensional scalar optimization problem $(P)$.

Proof. Contrary to the result, we assume that $(\bar{a}, \bar{c})$ is not a weak robust optimal solution to multi-dimensional scalar optimization problem $(P)$. Then, there exists another point $(\tilde{a}, \tilde{c}) \in D$ such that

$$
\int_{\Theta} \max _{w \in W} f(\tilde{\pi}, w) d t<\int_{\Theta} \max _{w \in W} f(\bar{\pi}, w) d t .
$$

Since $\max _{w \in W} f(\pi, w)=f(\pi, \bar{w})$, we obtain

$$
\int_{\Theta} f(\tilde{\pi}, \bar{w}) d t<\int_{\Theta} f(\bar{\pi}, \bar{w}) d t .
$$

By assumption, since $\int_{\Theta} f(\pi, \bar{w}) d t$ is a convex functional at $(\bar{a}, \bar{c})$, we have

$$
\begin{gathered}
\int_{\Theta}\{f(\tilde{\pi}, \bar{w})-f(\bar{\pi}, \bar{w})\} d t \geqq \int_{\Theta} f_{a}(\bar{\pi}, \bar{w})(\tilde{a}-\bar{a}) d t+\int_{\Theta} f_{a_{\sigma}}(\bar{\pi}, \bar{w})\left(\tilde{a}_{\sigma}-\bar{a}_{\sigma}\right) d t \\
+\frac{1}{n(\alpha, \beta)} \int_{\Theta} f_{a_{\alpha \beta}}(\bar{\pi}, \bar{w})\left(\tilde{a}_{\alpha \beta}-\bar{a}_{\alpha \beta}\right) d t+\int_{\Theta} f_{\mathcal{c}}(\bar{\pi}, \bar{w})(\tilde{c}-\bar{c}) d t
\end{gathered}
$$


which along with the inequality (10), yield

$$
\begin{gathered}
\int_{\Theta} f_{a}(\bar{\pi}, \bar{w})(\tilde{a}-\bar{a}) d t+\int_{\Theta} f_{a_{\sigma}}(\bar{\pi}, \bar{w})\left(\tilde{a}_{\sigma}-\bar{a}_{\sigma}\right) d t \\
+\frac{1}{n(\alpha, \beta)} \int_{\Theta} f_{a_{\alpha \beta}}(\bar{\pi}, \bar{w})\left(\tilde{a}_{\alpha \beta}-\bar{a}_{\alpha \beta}\right) d t+\int_{\Theta} f_{c}(\bar{\pi}, \bar{w})(\tilde{c}-\bar{c}) d t<0,
\end{gathered}
$$

equivalently with

$$
\begin{gathered}
\int_{\Theta} f_{a}(\bar{\pi}, \bar{w})(\tilde{a}-\bar{a}) d t+\int_{\Theta} f_{a_{\sigma}}(\bar{\pi}, \bar{w})\left(\tilde{a}_{\sigma}-\bar{a}_{\sigma}\right) d t \\
+\frac{1}{n(\alpha, \beta)} \int_{\Theta} f_{a_{\alpha \beta}}(\bar{\pi}, \bar{w})\left(\tilde{a}_{\alpha \beta}-\bar{a}_{\alpha \beta}\right) d t+\int_{\Theta} f_{c}(\bar{\pi}, \bar{w})(\tilde{c}-\bar{c}) d t \\
<\int_{\Theta} f_{a}(\bar{\pi}, \bar{w})(\bar{a}-\bar{a}) d t+\int_{\Theta} f_{a_{\sigma}}(\bar{\pi}, \bar{w})\left(\bar{a}_{\sigma}-\bar{a}_{\sigma}\right) d t \\
+\frac{1}{n(\alpha, \beta)} \int_{\Theta} f_{a_{\alpha \beta}}(\bar{\pi}, \bar{w})\left(\bar{a}_{\alpha \beta}-\bar{a}_{\alpha \beta}\right) d t+\int_{\Theta} f_{c}(\bar{\pi}, \bar{w})(\bar{c}-\bar{c}) d t .
\end{gathered}
$$

The above inequality can be rewritten as

$$
\begin{gathered}
\int_{\Theta} \max _{w \in W}\left[f_{a}(\bar{\pi}, w)(\tilde{a}-\bar{a})+f_{a_{\sigma}}(\bar{\pi}, w)\left(\tilde{a}_{\sigma}-\bar{a}_{\sigma}\right)\right. \\
\left.+\frac{1}{n(\alpha, \beta)} f_{a_{\alpha \beta}}(\bar{\pi}, w)\left(\tilde{a}_{\alpha \beta}-\bar{a}_{\alpha \beta}\right)+f_{c}(\bar{\pi}, w)(\tilde{c}-\bar{c})\right] d t \\
<\int_{\Theta} \max _{w \in W}\left[f_{a}(\bar{\pi}, w)(\bar{a}-\bar{a})+f_{a_{\sigma}}(\bar{\pi}, w)\left(\bar{a}_{\sigma}-\bar{a}_{\sigma}\right)\right. \\
\left.+\frac{1}{n(\alpha, \beta)} f_{a_{\alpha \beta}}(\bar{\pi}, w)\left(\bar{a}_{\alpha \beta}-\bar{a}_{\alpha \beta}\right)+f_{c}(\bar{\pi}, w)(\bar{c}-\bar{c})\right] d t,
\end{gathered}
$$

which contradicts our assumption that $(\bar{a}, \bar{c})$ is a weak robust optimal solution to the modified multi-dimensional scalar optimization problem $(P)_{(\bar{a}, \bar{c})}$. Hence, the proof is completed.

Remark 3. As can be easily seen, to derive the equivalence between the original problem $(P)$ and its associated modified problem $(P)_{(\bar{a}, \bar{c})}$, we imposed the convexity assumption only on the constraint functionals; whereas, to establish the converse of Theorem 2, it is sufficient to consider only the convexity hypothesis of the objective functional.

\section{Conclusions}

In this paper, for a given class of constrained robust optimization problems, named $(P)$, we have introduced and investigated an auxiliary (modified) robust optimization problem. More specifically, by considering multiple integral cost functionals and mixed constraints involving second-order partial derivatives and data uncertainty, we have formulated and proved some equivalence results for the considered control problems. For this aim, we used the concept of convexity associated with multiple integral functionals and the notion of a normal weak robust optimal solution.

Some developments of the results presented in this paper, which will be investigated in future papers, are provided by the study of the saddle-point optimality criteria and the associated (Wolfe, Mond-Weir, mixed) duality theory for the considered class of control problems.

Funding: This research received no external funding.

Institutional Review Board Statement: Not applicable. 
Informed Consent Statement: Not applicable.

Data Availability Statement: Not applicable.

Conflicts of Interest: The author declares no conflict of interest.

\section{References}

1. Mititelu, S.. Optimality and duality for invex multi-dimensional control problems with mixed constraints. J. Adv. Math. Stud. 2009, 2, 25-34.

2. Treanţă, S. Multiobjective fractional variational problem on higher-order jet bundles. Commun. Math. Stat. 2016, 4, 323-340. [CrossRef]

3. Olteanu, O.; Treanţă, S. Convexity, Optimization and Approximation, with Some Applications; LAP Lambert Academic Publishing: Saarbrücken, Germany, 2018; ISBN: 978-613-9-87683-9.

4. Mititelu, S..; Treanţă, S. Efficiency conditions in vector control problems governed by multiple integrals. J. Appl. Math. Comput. 2018, 57, 647-665. [CrossRef]

5. Jayswal, A.; Antczak, T.; Jha, S. On equivalence between a variational problem and its modified variational problem with the $\eta$-objective function under invexity. Int. Trans. Opt. Res. 2019, 26, 2053-2070. [CrossRef]

6. Treanţă, S. Saddle-point optimality criteria in modified variational control problems with PDE constraints. Optim. Control Appl. Meth. 2020, 41, 1160-1175. [CrossRef]

7. Treanţă, S. On Modified Interval-Valued Variational Control Problems with First-Order PDE Constraints. Symmetry 2020, $12,472$. [CrossRef]

8. Treanţă, S. On a modified optimal control problem with first-order PDE constraints and the associated saddle-point optimality criterion. Eur. J. Control 2020, 51, 1-9. [CrossRef]

9. Treanţă, S. Saddle-point optimality criteria involving $(\rho, b, d)$-invexity and $(\rho, b, d)$-pseudoinvexity in interval-valued optimization problems. Int. J. Control 2021. [CrossRef]

10. Liu, X.W.; Yuan, Y.X. A robust algorithm for optimization with general equality and inequality constraints. SIAM J. Sci. Comput. 2000, 22, 517-534. [CrossRef]

11. Jeyakumar, V.; Wang, J.H.; Li, G. Lagrange multiplier characterizations of robust best approximations under constraint data uncertainty. J. Math. Anal. Appl. 2012, 393, 285-297. [CrossRef]

12. Wei, H.Z.; Chen, C.R.; Li, S.J. Characterizations for optimality conditions of general robust optimization problems. J. Optim. Theory Appl. 2018, 177, 835-856. [CrossRef]

13. Jayswal, P.A.; Arana-Jiménez, M. Robust saddle-point criteria for multi-dimensional optimization problems with data uncertainty. Int. J. Control 2020. [CrossRef]

14. Sun, X.K.; Teo, K.L.; Zeng, J.; Guo, X.L. On approximate solutions and saddle point theorems for robust convex optimization. Optim. Lett. 2020, 14, 1711-1730. [CrossRef]

15. Treanţă, S. Efficiency in uncertain variational control problems. Neural Comput. Appl. 2021, 33, 5719-5732. [CrossRef]

16. Lu, Z.; Zhu, Y.; Lu, Q. Stability analysis of nonlinear uncertain fractional differential equations with Caputo derivative. Fractals 2021, 29, 2150057. [CrossRef]

17. Affane, D.; Yarou, M.F. General second order functional differential inclusion driven by the sweeping process with subsmooth sets. J. Nonlinear Funct. Anal. 2020, 2020, 26.

18. Cernea, A. On the mild solutions of a class of second-order integro-differential inclusions. J. Nonlinear Var. Anal. 2019, 3, 247-256.

19. Treanţă, S. On a Dual Pair of Multiobjective Interval-Valued Variational Control Problems. Mathematics 2021, 9, 893. [CrossRef]

20. Treanţă, S. Duality Theorems for $(\rho, \psi, d)$-Quasiinvex Multiobjective Optimization Problems with Interval-Valued Components. Mathematics 2021, 9, 894. [CrossRef]

21. Saunders, D.J. The Geometry of Jet Bundles; London Math. Soc. Lecture Notes Series, 142; Cambridge Univ. Press: Cambridge, UK, 1989.

22. Treanţă, S. Constrained variational problems governed by second-order Lagrangians. Appl. Anal. 2020, 99, 1467-1484. [CrossRef] 